\title{
Spectroscopic parameters for a sample of metal-rich solar-type stars ${ }^{\star}, \star \star$
}

\author{
S. G. Sousa ${ }^{1,2,6}$, N. C. Santos ${ }^{1,3,4}$, G. Israelian ${ }^{5}$, M. Mayor $^{3}$, and M. J. P. F. G. Monteiro ${ }^{2,6}$ \\ ${ }^{1}$ Centro de Astronomia e Astrofísica da Universidade de Lisboa, Observatório Astronómico de Lisboa, Tapada da Ajuda, \\ 1349-018 Lisboa, Portugal \\ e-mail: sousasag@astro.up.pt \\ 2 Centro de Astrofísica da Universidade do Porto, Rua das Estrelas, 4150-762 Porto, Portugal \\ 3 Observatoire de Genève, 51 Ch. des Mailletes, 1290 Sauverny, Switzerland \\ ${ }^{4}$ Centro de Geofísica de Évora, Colégio Luis Antonio Verney, Évora, Portugal \\ 5 Instituto de Astrofísica de Canarias, 38200 La Laguna, Tenerife, Spain \\ 6 Departamento de Matemática Aplicada, Faculdade de Ciências da Universidade do Porto, Portugal
}

Received 22 May 2006 / Acccepted 20 July 2006

\section{ABSTRACT}

\begin{abstract}
Aims. To date, metallicity is the only parameter of a star that appears to clearly correlate with the presence of planets and their properties. To check for new correlations between stars and the existence of an orbiting planet, we determine accurate stellar parameters for several metal-rich solar-type stars. The purpose is to fill the gap of the comparison sample presented in previous works in the high metal-content regime.

Methods. The stellar parameters were determined using an LTE analysis based on equivalent widths $(E W)$ of iron lines and by imposing excitation and ionization equilibrium. We also present a first step in determining these stellar parameters in an automatic manner by using the code DAOSPEC for the $E W$ determination.

Results. Accurate stellar parameters and metallicities are obtained for our sample composed of 64 high metal-content stars not known to harbor any planet. This sample will in the future give us the possibility of better exploring the existence of differences in the chemical abundances between planet-host stars and stars without known planets in the metal-rich domain. We also report stellar parameters for some recently discovered planet-host stars. Finally, we present an empirical calibration for DAOSPEC based on the comparison between its $E W$ measurements and the standard "hand made" measurements for the FEROS sample presented in this paper.
\end{abstract}

Key words. stars: abundances - stars: fundamental parameters - stars: planetary systems - stars: planetary systems: formation

\section{Introduction}

There are about 180 known stars that host planets at the moment. One well established characteristic of these stars is that they are very rich in metal when compared with stars not known to host any planet (Gonzalez 1997, 1998; Santos et al. 2001). At the present time the observed dependence of planet frequency on stellar metallicity is the only well-established correlation known to exist between the presence of planets, their properties, and stellar characteristics. But other suggested connections between planetary properties and the metal content of the host stars exist, such as the possible period-metallicity correlation (Sozzetti 2004; Santos et al. 2006) and the correlation between the heavyelement content of transiting planets and the stellar metallicity (Guillot et al. 2006).

Interesting is that there are hints that the well-established correlation between stellar metallicity and and the presence of a planet may only be present for stars hosting giant planets, while, for the stars orbited by very low-mass Neptune-like planets, the

* Based on observations collected at La Silla Observatory, ESO, Chile, with the FEROS spectrograph at the 2.2-m MPI/ESO telescope (074.C-0135), with the CORALIE spectrograph at the 1.2-m Euler Swiss Telescope, and with the SARG spectrograph at the TNG telescope, operated at the island of La Palma.

$\star \star$ Tables 2-4 are also available in electronic form at the CDS via anonymous ftp to cdsarc.u-strasbg.fr (130.79.128.5) or via http://cdsweb.u-strasbg.fr/cgi-bin/qcat?J/A+A/458/873 correlation may not be present (Udry et al. 2006). This conclusion, which is compatible with the most recent models of planet formation (C. Mordasini, private communication), was the output of the continuous follow-up study of the chemical abundances of planet-host stars, and it illustrates how important it is to continue to enlarge the samples.

Current studies seem to indicate that the excess metallicity found for stars harboring giant planets has a primordial origin; i.e., that the high metal content of the stars was common to the cloud of gas and dust that originated the star-planet system (Pinsonneault et al. 2001; Santos et al. 2001, 2003). However, the debate about the origin of this metallicity excess is still not settled (Vauclair 2004). It was already shown that although it is not the major source, element pollution may indeed occur (Israelian et al. 2001, 2003; Laws et al. 2003). The search for trends in metallicity with condensation temperature got carried away with the main results that they have not found any significant differences between planet-host stars and a comparison sample of stars. This is consistent with a "primordial" origin to the metal excess in planet-host stars (Ecuvillon et al. 2006a; Gonzalez 2006; Sozzetti et al. 2006). However, there seems to be a few stars found by Ecuvillon et al. (2006a) that show significant trends.

To understand how much "pollution" phenomena might have been able to change the metal content of the stellar atmospheres, we need to continue following the chemical abundances for several elements on planet-host stars as new planets are found, 
as well as for stars with no planets found, to see if there are any significant differences.

On the other hand, the studing element abundances may shed some new light on the process of planetary formation. Robinson et al. (2006) found some statistical evidence of silicon and nickel enrichment in planet-host stars. This putative enhancement, not observed however by other authors (cf. Gilli et al. 2006), may have to do with the influence that different types of elements may have on the process of planetary formation. Such scenarios have been proposed by e.g. Gaidos (2000).

The main results of a uniform study, concerning the metallicity of planet-host stars, were presented in Santos et al. (2000, 2001, 2003, 2004, 2005, Papers I, II, III, IV, V, respectively). In this paper we present results for a new sample of stars not known to harbor any planet. The purpose is to increase the comparison sample of stars with high metal content, since only a relatively small sample of metal-rich $([\mathrm{Fe} / \mathrm{H}] \geq 0.20)$ comparison field stars without known planets had been analyzed.

In a future paper this sample will be used to analyze elemental abundances with the goal of extending previous works in this field (Bodaghee et al. 2003; Beirão et al. 2005; Ecuvillon et al. 2004a,b, 2006b; Gilli et al. 2006). With this high metalcontent sample we will be able to search for new possible correlations between planet-host and "single" stars by comparing abundances for several other elements in the high $[\mathrm{Fe} / \mathrm{H}]$ regime.

We also present in this paper stellar parameters for some recently discovered planet-host stars. The purpose is to update the series of Papers I-V that, together with this one, will have the largest set of the stellar parameters available to date for planethost stars while using the same uniform analysis.

Finally, we compare the measurements of line $E W$ obtained in the regular way with the ones obtained by a new automatic code named DAOSPEC (Pancino \& Stetson 2006). The impact on the derived stellar parameters is discussed.

\section{Sample and observations}

The stars in this sample belong to the CORALIE southern planet search program (Udry et al. 2000), have spectral types between $\mathrm{F}$ and $\mathrm{K}$, and were not announced as harboring any planetary companion. From these stars we selected the ones with at least 3 measurements of radial velocity spanning at least one year and ones that did not present any significant variation that could correspond to the RV signal induced by a low-mass companion. The majority of the stars have a typical rms on the radial velocity lower than $10 \mathrm{~m} / \mathrm{s}$, and for a few of them this value is between $10 \mathrm{~m} / \mathrm{s}$ and $20 \mathrm{~m} / \mathrm{s}$. Moreover, we chose the ones that have $[\mathrm{Fe} / \mathrm{H}] \geq 0.1$ determined using the CORALIE cross-correlation function as presented in the work of Santos et al. (2002).

The majority of the spectra were collected with the FEROS spectrograph (2.2 m ESO/MPI telescope, La Silla, Chile) on October 2004 (program 074.C-0135). Data reduction was done using the FEROS pipeline, and the wavelength calibration used the spectrum of a Th-Ar lamp. The final spectra have a resolution $R=\Delta \lambda / \lambda=48000$ and $S / N$ between $\sim 200$ and 400 . More spectra were obtained with a resolution of 57000 using the SARG spectrograph at the TNG telescope (La Palma Observatory, Spain). For these the data was reducted using $\mathrm{IRAF}^{1}$ tools in the echelle package.

${ }^{1}$ IRAF is distributed by National Optical Astronomy Observatories, operated by the Association of Universities for Research in Astronomy, Inc., under contract with the National Science Foundation, USA.
Table 1. Atomic parameters and measured solar equivalent widths for the 4 new Fe II lines used in this paper and not used in our previous papers.

\begin{tabular}{lccc}
\hline \hline$\lambda(\AA)$ & $\chi_{l}$ & $\log g f$ & $E W_{\odot}(\mathrm{m} \AA)$ \\
\hline 5325.55 & 3.22 & -3.12 & 41.5 \\
5414.07 & 3.22 & -3.54 & 26.6 \\
5425.25 & 3.19 & -3.16 & 41.8 \\
6456.38 & 3.90 & -2.10 & 62.9 \\
\hline
\end{tabular}

Some planet-host stars not previously studied by our team were also observed. The spectra for these were collected using 4 different instruments: FEROS, SARG, the CORALIE spectrograph $(R=50000)$ at the $1.2-\mathrm{m}$ Swiss telescope (La Silla, Chile) and the HARPS spectrograph $(R=110000)$ at the $3.6-\mathrm{m}$ ESO (La Silla, Chile).

\section{Spectroscopic analysis and stellar parameters}

The spectroscopic analysis was done in LTE using the 2002 version of the code MOOG $^{2}$ (Sneden 1973) and a grid of Kurucz Atlas plane-parallel model atmospheres (Kurucz 1993).

Stellar parameters and metallicities were derived as in Santos et al. (2004), based on the $E W$ of Fe I and Fe II weak lines, by imposing excitation and ionization equilibrium. Four new Fe II lines shown in Table 1 were added to the lists presented in Santos et al. (2004) and James et al. (2006).

The $E W$ were measured using IRAF "splot" and "bplot" routines within the echelle package. The stellar parameters are determined using an Downhill simplex method (Press et al. 1992) to find the best solution for the measured $E W$. The results of this analysis are presented in Tables 2 and 3. The results for the planet-host stars are presented in Table 4, but some of the parameters for these stars have already been presented elsewhere.

In Table 4 we also present metallicities for HD 34445 and HD 196885, although these stars are still to be confirmed as planet-host stars, since no announcement paper has been published. The reason they appear in this table is that they are presented as planet-hosts in the extra-solar planets encyclope$\mathrm{dia}^{3}$. The errors in $T_{\text {eff }}, \log g, \xi_{t}$, and $[\mathrm{Fe} / \mathrm{H}]$ were derived as in Paper IV. The procedure is described in detail in Gonzalez \& Lambert (1996) (except for the case of $\log g$, in this case we divided the dispersion of the Fe II lines by the square root of the number of lines used). The values are typically on the order of $50 \mathrm{~K}, 0.1 \mathrm{dex}, 0.1 \mathrm{~km} \mathrm{~s}^{-1}$, and $0.05 \mathrm{dex}$, respectively. Higher uncertainties are found for lower temperature stars.

The masses presented in the tables were determined from an interpolation of the theoretical isochrones of Schaller et al. (1992) and Schaerer et al. (1993a,b), using $M_{V}$ obtained through the Hipparcos parallaxes (ESA 1997), a bolometric correction (BC) from Flower (1996) and $T_{\text {eff }}$ obtained from the spectroscopy. We adopted a typical uncertainty of $0.05 M_{\odot}$ for the masses. In some cases, no mass estimates are presented, since these involve large extrapolations of the isochrones.

We also derived the "trigonometric" surface gravities for our stars from the estimated masses, the spectroscopic temperature, and $\mathrm{BC}$, using the same basic formula as was presented in Santos et al. (2004). As seen in previous works we also found that the two surface-gravity estimates are correlated well (see Fig. 1).

\footnotetext{
2 The source code of MOOG2002 can be downloaded at http://verdi.as.utexas. edu/moog.html

3 http://www . exoplanets.eu
} 
Table 2. Stellar parameters for our sample for HD numbers between 1 and 60000.

\begin{tabular}{|c|c|c|c|c|c|c|c|c|c|}
\hline $\begin{array}{l}\text { HD } \\
\text { number }\end{array}$ & $\begin{array}{l}T_{\text {eff }} \\
{[\mathrm{K}]}\end{array}$ & $\begin{array}{l}\log g_{\mathrm{spec}} \\
{\left[\mathrm{cm} \mathrm{s}^{-2}\right]}\end{array}$ & $\begin{array}{c}\xi_{\mathrm{t}} \\
{\left[\mathrm{km} \mathrm{s}^{-1}\right]}\end{array}$ & 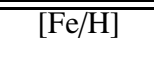 & $\bar{~} N(\mathrm{Fe}$ I,Fe II $)$ & 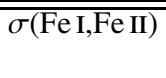 & 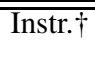 & $\begin{array}{l}\text { Mass } \\
{\left[M_{\odot}\right]}\end{array}$ & $\begin{array}{l}\log g_{\text {hipp }} \\
{\left[\mathrm{cm} \mathrm{s}^{-2}\right]}\end{array}$ \\
\hline HD 7199 & $5426 \pm 52$ & $4.41 \pm 0.07$ & $1.01 \pm 0.07$ & $0.39 \pm 0.06$ & 36,13 & $0.06,0.09$ & [1] & 0.94 & 4.44 \\
\hline HD 7570 & $6140 \pm 41$ & $4.39 \pm 0.16$ & $1.50 \pm 0.08$ & $0.18 \pm 0.05$ & 35,6 & $0.04,0.05$ & [3] & 1.20 & 4.36 \\
\hline HD 7570 & $6198 \pm 39$ & $4.49 \pm 0.02$ & $1.40 \pm 0.07$ & $0.24 \pm 0.05$ & 38,15 & $0.04,0.06$ & [1] & 1.25 & 4.39 \\
\hline HD 7570 & 6169 & 4.44 & 1.45 & 0.21 & & & avg. & 1.23 & 4.38 \\
\hline HD 7727 & $6131 \pm 41$ & $4.34 \pm 0.02$ & $1.18 \pm 0.07$ & $0.16 \pm 0.05$ & 38,15 & $0.05,0.07$ & [1] & 1.19 & 4.38 \\
\hline HD 8326 & $4914 \pm 63$ & $4.30 \pm 0.11$ & $0.90 \pm 0.09$ & $0.10 \pm 0.07$ & 36,12 & $0.06,0.13$ & [1] & 0.68 & 4.49 \\
\hline HD 8389A & $5378 \pm 84$ & $4.50 \pm 0.12$ & $1.09 \pm 0.09$ & $0.47 \pm 0.08$ & 36,13 & $0.07,0.12$ & [1] & 0.96 & 4.48 \\
\hline HD 9562 & $5937 \pm 36$ & $4.13 \pm 0.02$ & $1.34 \pm 0.05$ & $0.26 \pm 0.05$ & 37,16 & $0.04,0.07$ & [1] & 1.28 & 4.03 \\
\hline HD 9782 & $6023 \pm 36$ & $4.40 \pm 0.02$ & $1.11 \pm 0.06$ & $0.15 \pm 0.05$ & 39,16 & $0.04,0.06$ & [1] & 1.14 & 4.40 \\
\hline HD 10002 & $5354 \pm 54$ & $4.43 \pm 0.07$ & $0.96 \pm 0.07$ & $0.26 \pm 0.06$ & 37,13 & $0.05,0.08$ & [1] & 0.91 & 4.51 \\
\hline HD 10180 & $5912 \pm 24$ & $4.33 \pm 0.01$ & $1.10 \pm 0.03$ & $0.13 \pm 0.04$ & 37,16 & $0.03,0.06$ & [1] & 1.07 & 4.34 \\
\hline HD 10226 & $6039 \pm 28$ & $4.48 \pm 0.02$ & $1.16 \pm 0.04$ & $0.22 \pm 0.04$ & 38,15 & $0.03,0.05$ & [1] & 1.19 & 4.47 \\
\hline HD 11226 & $6099 \pm 32$ & $4.31 \pm 0.01$ & $1.30 \pm 0.06$ & $0.09 \pm 0.04$ & 38,15 & $0.04,0.04$ & [1] & 1.18 & 4.26 \\
\hline HD 12058 & $4804 \pm 111$ & $4.39 \pm 0.23$ & $1.18 \pm 0.17$ & $0.10 \pm 0.12$ & 36,9 & $0.11,0.41$ & [1] & 0.82 & 4.84 \\
\hline HD 13043A & $5934 \pm 21$ & $4.33 \pm 0.01$ & $1.22 \pm 0.03$ & $0.14 \pm 0.03$ & 38,15 & $0.03,0.05$ & [1] & 1.15 & 4.25 \\
\hline HD 13386 & $5226 \pm 56$ & $4.28 \pm 0.09$ & $0.88 \pm 0.07$ & $0.26 \pm 0.06$ & 36,14 & $0.06,0.11$ & [1] & 0.84 & 4.46 \\
\hline HD 16270 & $4891 \pm 120$ & $4.43 \pm 0.22$ & $1.19 \pm 0.17$ & $0.16 \pm 0.12$ & 36,9 & $0.11,0.17$ & [1] & 0.84 & 4.73 \\
\hline HD 16417 & $5876 \pm 22$ & $4.22 \pm 0.01$ & $1.24 \pm 0.03$ & $0.20 \pm 0.03$ & 37,15 & $0.03,0.04$ & [1] & 1.23 & 4.14 \\
\hline HD 20201 & $6064 \pm 34$ & $4.43 \pm 0.02$ & $1.17 \pm 0.05$ & $0.19 \pm 0.05$ & 38,15 & $0.04,0.06$ & [1] & 1.19 & 4.45 \\
\hline HD 21197 & $4894 \pm 202$ & $4.63 \pm 0.40$ & $1.35 \pm 0.31$ & $0.18 \pm 0.17$ & 38,9 & $0.16,0.51$ & [1] & 0.87 & 4.85 \\
\hline HD 26151 & $5388 \pm 56$ & $4.31 \pm 0.08$ & $1.02 \pm 0.07$ & $0.28 \pm 0.07$ & 39,13 & $0.06,0.06$ & [1] & 0.88 & 4.37 \\
\hline HD 30306 & $5580 \pm 33$ & $4.34 \pm 0.04$ & $0.93 \pm 0.04$ & $0.26 \pm 0.04$ & 38,15 & $0.04,0.05$ & [1] & 0.96 & 4.36 \\
\hline HD 30562 & $5970 \pm 37$ & $4.20 \pm 0.02$ & $1.20 \pm 0.05$ & $0.32 \pm 0.05$ & 38,13 & $0.04,0.05$ & [1] & 1.29 & 4.15 \\
\hline HD 32147 & $4705 \pm 51$ & $4.44 \pm 0.31$ & $0.60 \pm 0.17$ & $0.30 \pm 0.08$ & 35,4 & $0.07,0.15$ & [3] & - & - \\
\hline HD 32147 & $4937 \pm 136$ & $4.33 \pm 0.26$ & $1.02 \pm 0.17$ & $0.27 \pm 0.12$ & 37,11 & $0.10,0.26$ & [1] & 0.87 & 4.67 \\
\hline HD 32147 & 4821 & 4.38 & 0.81 & 0.29 & & & avg. & - & - \\
\hline HD 33214 & $5180 \pm 74$ & $4.40 \pm 0.11$ & $1.10 \pm 0.10$ & $0.17 \pm 0.08$ & 39,14 & $0.08,0.13$ & [1] & 0.90 & 4.62 \\
\hline HD 36152 & $5790 \pm 25$ & $4.46 \pm 0.02$ & $1.02 \pm 0.04$ & $0.11 \pm 0.04$ & 39,16 & $0.03,0.07$ & [1] & 1.07 & 4.52 \\
\hline HD 36553 & $6103 \pm 46$ & $3.85 \pm 0.03$ & $1.61 \pm 0.07$ & $0.41 \pm 0.06$ & 37,15 & $0.05,0.09$ & [1] & 1.71 & 3.75 \\
\hline HD 37986 & $5586 \pm 42$ & $4.38 \pm 0.05$ & $1.04 \pm 0.05$ & $0.35 \pm 0.05$ & 38,14 & $0.05,0.05$ & [1] & 1.01 & 4.49 \\
\hline HD 39833 & $5901 \pm 34$ & $4.48 \pm 0.02$ & $1.15 \pm 0.05$ & $0.20 \pm 0.05$ & 39,15 & $0.04,0.04$ & [1] & 1.09 & 4.32 \\
\hline HD 43745 & $6086 \pm 28$ & $3.98 \pm 0.01$ & $1.42 \pm 0.05$ & $0.14 \pm 0.04$ & 38,15 & $0.03,0.05$ & [1] & 1.42 & 4.00 \\
\hline HD 47186 & $5696 \pm 29$ & $4.39 \pm 0.03$ & $1.04 \pm 0.04$ & $0.27 \pm 0.04$ & 38,14 & $0.04,0.03$ & [1] & 1.01 & 4.38 \\
\hline HD 55693 & $5951 \pm 34$ & $4.41 \pm 0.02$ & $1.22 \pm 0.04$ & $0.32 \pm 0.04$ & 37,16 & $0.04,0.04$ & [1] & 1.15 & 4.36 \\
\hline HD 58895 & $5844 \pm 40$ & $4.01 \pm 0.03$ & $1.39 \pm 0.04$ & $0.37 \pm 0.05$ & 39,16 & $0.05,0.05$ & [1] & 1.45 & 3.95 \\
\hline
\end{tabular}

$\dagger$ The instruments used to obtain the spectra were: [1] 2.2-m ESO/FEROS, [2] TNG/SARG, [3] 1.2-m Swiss Telescope/CORALIE. Parameters from CORALIE for HD7570 and HD32147 belong to Paper IV and V, respectively.

The final $[\mathrm{Fe} / \mathrm{H}]$ distribution of the sample can be seen in Fig. 2. Figure 3 shows a comparison between the metallicity obtained through the cross-correlation function and the spectroscopic metallicity obtained in this work. From this figure we see that the former provides a good preliminary metallicity determination.

\section{Determining stellar parameters with the help of DAOSPEC}

The present discovery rate of planets means there is now a problem with the large amount of data to analyze. We need to analyze each planet-host in detail and also a significant and increasing amount of stars without planets hoping to find some relevant clues to the formation and evolution of the planets themselves. To face this problem we are trying to automate the spectroscopic stellar-parameter determination.

In this paper we used a standard technique based on the iron excitation and ionization balance of Fe I and Fe II lines. Using this method we needed to measure the equivalents widths of several iron lines in the stellar spectra. For this purpose we are testing DAOSPEC, a new automatic code developed by P. Stetson \& E. Pancino (2006 in preparation, http://cadcwww.hia.nrc.ca/stetson/daospec/).

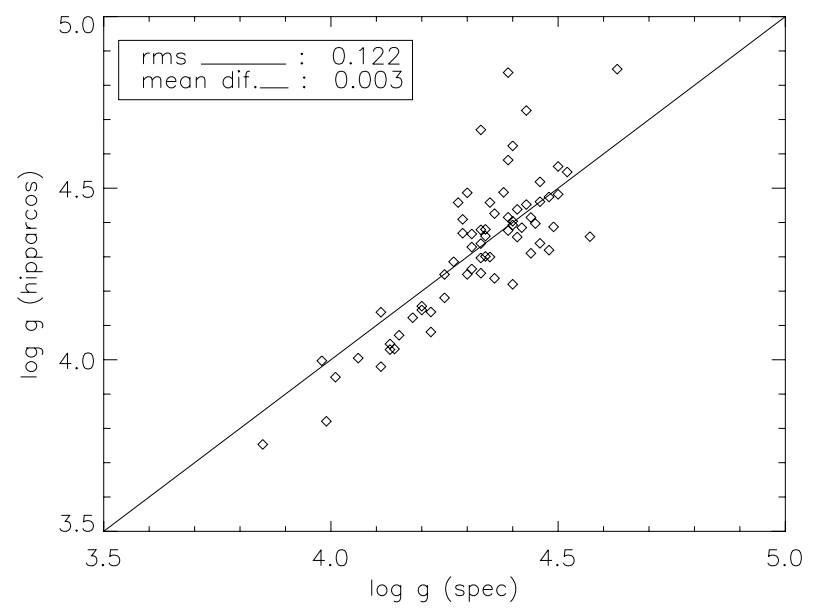

Fig. 1. Comparison of the spectroscopic and parallax-based surface gravities of our program stars for the stars presented in this paper. The solid line represents a 1:1 relation.

Among other tasks this code measures $E W$ in an automatic manner. 
Table 3. Stellar parameters for our sample for HD numbers from 60000 on.

\begin{tabular}{|c|c|c|c|c|c|c|c|c|c|}
\hline $\begin{array}{l}\text { HD } \\
\text { number }\end{array}$ & $\begin{array}{l}T_{\text {eff }} \\
{[\mathrm{K}]}\end{array}$ & $\begin{array}{l}\log g_{\text {spec }} \\
{\left[\mathrm{cm} \mathrm{s}^{-2}\right]}\end{array}$ & $\begin{array}{c}\xi_{\mathrm{t}} \\
{\left[\mathrm{km} \mathrm{s}^{-1}\right]}\end{array}$ & $\overline{[\overline{\mathrm{Fe}} / \mathrm{H}]}$ & $\overline{\mathrm{N}(\mathrm{Fe}}$ I,Fe II $)$ & 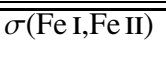 & $\overline{\text { Instr. } \dagger}$ & $\begin{array}{l}\text { Mass } \\
{\left[M_{\odot}\right]}\end{array}$ & $\begin{array}{l}\log g_{\text {hipp }} \\
{\left[\mathrm{cm} \mathrm{s}^{-2}\right]}\end{array}$ \\
\hline HD 66221 & $5655 \pm 33$ & $4.39 \pm 0.03$ & $1.05 \pm 0.04$ & $0.21 \pm 0.05$ & 39,15 & $0.04,0.04$ & [1] & 0.98 & 4.42 \\
\hline HD 67556 & $6281 \pm 53$ & $4.29 \pm 0.02$ & $1.23 \pm 0.09$ & $0.32 \pm 0.06$ & 37,15 & $0.05,0.09$ & [1] & 1.31 & 4.37 \\
\hline HD 73121 & $6092 \pm 39$ & $4.25 \pm 0.02$ & $1.32 \pm 0.07$ & $0.14 \pm 0.05$ & 38,16 & $0.04,0.05$ & [1] & 1.25 & 4.18 \\
\hline HD 73524 & $6012 \pm 50$ & $4.40 \pm 0.03$ & $1.11 \pm 0.07$ & $0.23 \pm 0.06$ & 39,15 & $0.05,0.08$ & [1] & 1.15 & 4.39 \\
\hline HD 74868 & $6239 \pm 54$ & $4.27 \pm 0.02$ & $1.41 \pm 0.10$ & $0.35 \pm 0.07$ & 36,16 & $0.06,0.07$ & [1] & 1.30 & 4.29 \\
\hline HD 78429 & $5786 \pm 31$ & $4.34 \pm 0.02$ & $1.14 \pm 0.04$ & $0.11 \pm 0.05$ & 39,16 & $0.04,0.04$ & [1] & 1.01 & 4.30 \\
\hline HD 81110A & $5818 \pm 38$ & $4.50 \pm 0.03$ & $1.14 \pm 0.05$ & $0.31 \pm 0.05$ & 39,15 & $0.05,0.03$ & [1] & 1.17 & 4.56 \\
\hline HD 85380 & $6108 \pm 32$ & $4.13 \pm 0.01$ & $1.46 \pm 0.05$ & $0.16 \pm 0.04$ & 38,15 & $0.04,0.05$ & [1] & 1.38 & 4.05 \\
\hline HD 124553 & $6125 \pm 52$ & $4.22 \pm 0.02$ & $1.30 \pm 0.08$ & $0.28 \pm 0.06$ & 33,16 & $0.05,0.05$ & [2] & 1.39 & 4.08 \\
\hline HD 125184 & $5714 \pm 22$ & $4.18 \pm 0.02$ & $1.12 \pm 0.03$ & $0.32 \pm 0.03$ & 34,15 & $0.03,0.06$ & [2] & 1.22 & 4.12 \\
\hline HD 144585 & $5908 \pm 27$ & $4.36 \pm 0.02$ & $1.17 \pm 0.03$ & $0.34 \pm 0.04$ & 36,16 & $0.03,0.04$ & [2] & 1.21 & 4.24 \\
\hline HD 154962 & $5827 \pm 28$ & $4.15 \pm 0.02$ & $1.25 \pm 0.03$ & $0.34 \pm 0.04$ & 35,16 & $0.03,0.06$ & [2] & 1.28 & 4.07 \\
\hline HD 156365 & $5870 \pm 32$ & $4.11 \pm 0.02$ & $1.27 \pm 0.04$ & $0.30 \pm 0.05$ & 36,16 & $0.04,0.07$ & [2] & 1.41 & 3.98 \\
\hline HD 163153 & $5654 \pm 37$ & $4.14 \pm 0.04$ & $1.21 \pm 0.04$ & $0.40 \pm 0.05$ & 36,14 & $0.04,0.05$ & [2] & 1.31 & 4.03 \\
\hline HD 163272 & $6096 \pm 19$ & $4.44 \pm 0.01$ & $1.27 \pm 0.03$ & $0.32 \pm 0.02$ & 36,16 & $0.02,0.07$ & [2] & 1.22 & 4.31 \\
\hline HD 165920 & $5346 \pm 48$ & $4.36 \pm 0.07$ & $0.82 \pm 0.06$ & $0.31 \pm 0.05$ & 34,12 & $0.04,0.09$ & [2] & 0.88 & 4.43 \\
\hline HD 183658 & $5833 \pm 19$ & $4.42 \pm 0.01$ & $1.09 \pm 0.03$ & $0.08 \pm 0.03$ & 38,14 & $0.02,0.02$ & [1] & 1.02 & 4.38 \\
\hline HD 189625 & $5882 \pm 29$ & $4.46 \pm 0.02$ & $1.07 \pm 0.04$ & $0.24 \pm 0.04$ & 35,16 & $0.03,0.06$ & [2] & 1.11 & 4.46 \\
\hline HD 190248 & $5614 \pm 34$ & $4.29 \pm 0.07$ & $1.10 \pm 0.04$ & $0.36 \pm 0.04$ & 38,8 & $0.04,0.04$ & [3] & 1.02 & 4.31 \\
\hline HD 190248 & $5638 \pm 40$ & $4.33 \pm 0.04$ & $1.08 \pm 0.05$ & $0.39 \pm 0.05$ & 38,15 & $0.05,0.04$ & [1] & 1.02 & 4.30 \\
\hline HD 190248 & 5626 & 4.31 & 1.09 & 0.38 & & & avg. & 1.02 & 4.31 \\
\hline HD 192310 & $5069 \pm 49$ & $4.38 \pm 0.19$ & $0.79 \pm 0.07$ & $-0.01 \pm 0.05$ & 36,6 & $0.05,0.09$ & [3] & 0.72 & 4.47 \\
\hline HD 192310 & $5166 \pm 52$ & $4.39 \pm 0.08$ & $0.93 \pm 0.07$ & $0.04 \pm 0.06$ & 38,13 & $0.05,0.11$ & [1] & 0.83 & 4.58 \\
\hline HD 192310 & 5118 & 4.39 & 0.86 & 0.02 & & & avg. & 0.78 & 4.53 \\
\hline HD 199190 & $5946 \pm 25$ & $4.25 \pm 0.01$ & $1.22 \pm 0.03$ & $0.20 \pm 0.04$ & 38,15 & $0.03,0.05$ & [1] & 1.17 & 4.25 \\
\hline HD 199960 & $5999 \pm 31$ & $4.35 \pm 0.02$ & $1.21 \pm 0.04$ & $0.33 \pm 0.04$ & 38,14 & $0.04,0.03$ & [1] & 1.20 & 4.30 \\
\hline HD 203384 & $5546 \pm 38$ & $4.29 \pm 0.04$ & $0.97 \pm 0.05$ & $0.25 \pm 0.05$ & 33,12 & $0.04,0.10$ & [2] & 0.94 & 4.41 \\
\hline HD 204385 & $6022 \pm 24$ & $4.31 \pm 0.01$ & $1.22 \pm 0.04$ & $0.09 \pm 0.04$ & 38,15 & $0.03,0.04$ & [1] & 1.13 & 4.33 \\
\hline HD 205905 & $5979 \pm 31$ & $4.52 \pm 0.02$ & $1.12 \pm 0.06$ & $0.15 \pm 0.04$ & 34,12 & $0.03,0.04$ & [2] & 1.18 & 4.55 \\
\hline HD 212708 & $5722 \pm 37$ & $4.33 \pm 0.03$ & $1.13 \pm 0.04$ & $0.32 \pm 0.05$ & 39,16 & $0.04,0.05$ & [1] & 1.03 & 4.38 \\
\hline HD 214759 & $5459 \pm 42$ & $4.35 \pm 0.05$ & $0.97 \pm 0.05$ & $0.23 \pm 0.05$ & 39,14 & $0.05,0.06$ & [1] & 0.92 & 4.46 \\
\hline HD 216402 & $6400 \pm 50$ & $4.57 \pm 0.02$ & $1.65 \pm 0.12$ & $0.24 \pm 0.06$ & 32,14 & $0.05,0.07$ & [2] & 1.33 & 4.36 \\
\hline HD 221146 & $5969 \pm 31$ & $4.40 \pm 0.02$ & $1.23 \pm 0.05$ & $0.16 \pm 0.04$ & 34,15 & $0.03,0.04$ & [2] & 1.18 & 4.22 \\
\hline HD 221420 & $5864 \pm 43$ & $4.06 \pm 0.03$ & $1.35 \pm 0.05$ & $0.37 \pm 0.06$ & 38,15 & $0.05,0.05$ & [1] & 1.40 & 4.00 \\
\hline HD 222480 & $5848 \pm 24$ & $4.20 \pm 0.01$ & $1.23 \pm 0.03$ & $0.21 \pm 0.04$ & 37,14 & $0.03,0.03$ & [1] & 1.21 & 4.16 \\
\hline HD 223171 & $5828 \pm 28$ & $4.11 \pm 0.02$ & $1.20 \pm 0.04$ & $0.14 \pm 0.04$ & 39,14 & $0.03,0.05$ & [1] & 1.20 & 4.14 \\
\hline HD 224022 & $6134 \pm 46$ & $4.30 \pm 0.02$ & $1.52 \pm 0.09$ & $0.15 \pm 0.06$ & 38,16 & $0.05,0.07$ & [1] & 1.22 & 4.25 \\
\hline
\end{tabular}

$\dagger$ The instruments used to obtain the spectra were: [1] 2.2-m ESO/FEROS, [2] TNG/SARG, [3] 1.2-m Swiss Telescope/CORALIE. Parameters from CORALIE spectra for HD 192310 and HD 190248 were derived in Paper IV and V, respectively.

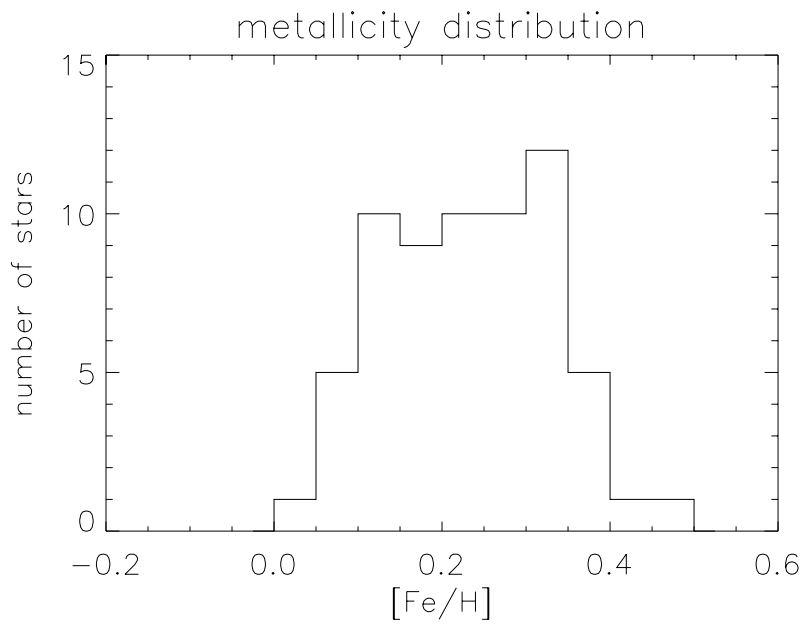

Fig. 2. Metalicity distribution of the presented sample.

\subsection{Testing DAOSPEC: using the solar spectrum}

Our main purpose is to test $E W$ measurements. Therefore we compare the measurements done with DAOSPEC with the "hand made" measurements obtained using the IRAF "splot" routine within the "echelle" package. As a first test we used the Kurucz Solar Atlas to produce several spectra with different levels of artificial noise and resolution. The noise was created using a Gaussian distribution, and the instrumental resolution was introduced using the "rotin3" routine in SYNSPEC 4 (Hubeny et al. 1994).

We then measured $E W$ of iron lines in two different regions of the solar spectrum. We chose 27 iron lines in the interval [4400 $\AA-4650 \AA]$ and 36 iron lines in the interval [6000 $6300 \AA]$. These iron lines were chosen so that they would not blended with other lines. The first wavelength interval is more line-populated than the second one.

In Fig. 4 we show the comparison of the measurements made by DAOSPEC ( $y$ axes) and the "hand made measurements" ( $x$ axes) for spectra with $S / N \sim 100$ and an artificial instrumental resolution $R=\Delta \lambda / \lambda \sim 50000$. In the figures we indicate the slope of the linear fit to the points, the number of lines identified by DAOSPEC, the rms and mean difference of the results

\footnotetext{
${ }^{4}$ http://tlusty.gsfc.nasa.gov/index.html
} 
Table 4. Stellar parameters for some planet-host stars.

\begin{tabular}{|c|c|c|c|c|c|c|c|c|c|}
\hline $\begin{array}{l}\text { HD } \\
\text { number }\end{array}$ & $\begin{array}{l}T_{\text {eff }} \\
{[\mathrm{K}]}\end{array}$ & $\begin{array}{l}\log g_{\text {spec }} \\
{\left[\mathrm{cm} \mathrm{s}^{-2}\right]}\end{array}$ & $\begin{array}{c}\xi_{\mathrm{t}} \\
{\left[\mathrm{km} \mathrm{s}^{-1}\right]}\end{array}$ & {$[\mathrm{Fe} / \mathrm{H}]$} & $\overline{\mathrm{N}(\mathrm{Fe} \text { I, Fe II })}$ & $\sigma(\mathrm{Fe} \mathrm{I}, \mathrm{Fe} \mathrm{II})$ & Ref. $\dagger$ & $\begin{array}{l}\text { Mass } \\
{\left[M_{\odot}\right]}\end{array}$ & $\begin{array}{l}\log g_{\text {hipp }} \\
{\left[\mathrm{cm} \mathrm{s}^{-2}\right]}\end{array}$ \\
\hline HD 4308 & $5685 \pm 13$ & $4.49 \pm 0.01$ & $1.08 \pm 0.03$ & $-0.31 \pm 0.01$ & 35,8 & $0.01,0.02$ & {$[\mathrm{a}][4]$} & 0.83 & 4.34 \\
\hline HD 11964A & $5372 \pm 35$ & $3.99 \pm 0.04$ & $1.09 \pm 0.04$ & $0.14 \pm 0.05$ & 38,15 & $0.04,0.05$ & {$[1]$} & 0.91 & 3.82 \\
\hline HD 11977 & $5020 \pm 30$ & $2.86 \pm 0.04$ & $1.46 \pm 0.03$ & $-0.09 \pm 0.06$ & 36,11 & $0.05,0.05$ & [3] & 2.31 & 2.78 \\
\hline HD 13189 & $4337 \pm 133$ & $1.83 \pm 0.31$ & $1.99 \pm 0.12$ & $-0.39 \pm 0.19$ & 34,10 & $0.18,0.16$ & [2] & 4.97 & 0.95 \\
\hline HD 28185 & $5656 \pm 44$ & $4.45 \pm 0.08$ & $1.01 \pm 0.06$ & $0.22 \pm 0.05$ & 38,6 & $0.05,0.03$ & {$[\mathrm{~b}][3]$} & 0.98 & 4.34 \\
\hline HD 28185 & $5710 \pm 30$ & $4.44 \pm 0.03$ & $1.08 \pm 0.04$ & $0.25 \pm 0.04$ & 37,14 & $0.03,0.04$ & [1] & 1.01 & 4.41 \\
\hline HD 28185 & 5683 & 4.44 & 1.04 & 0.24 & & & avg. & 1.00 & 4.38 \\
\hline HD 34445 & $5915 \pm 35$ & $4.30 \pm 0.02$ & $1.11 \pm 0.04$ & $0.24 \pm 0.04$ & 38,13 & $0.04,0.04$ & [3] & 1.17 & 4.25 \\
\hline HD 39091 & $5991 \pm 27$ & $4.42 \pm 0.10$ & $1.24 \pm 0.04$ & $0.10 \pm 0.04$ & 38,7 & $0.03,0.04$ & {$[\mathrm{~b}][1]$} & 1.10 & 4.38 \\
\hline HD 39091 & $6027 \pm 30$ & $4.45 \pm 0.02$ & $1.34 \pm 0.06$ & $0.11 \pm 0.04$ & 39,16 & $0.03,0.05$ & [1] & 1.12 & 4.40 \\
\hline HD 39091 & 6009 & 4.44 & 1.29 & 0.10 & & & avg. & 1.11 & 4.39 \\
\hline HD 50499 & $6056 \pm 47$ & $4.29 \pm 0.03$ & $1.23 \pm 0.06$ & $0.39 \pm 0.06$ & 39,13 & $0.05,0.07$ & [3] & 1.26 & 4.25 \\
\hline HD 69830 & $5385 \pm 20$ & $4.37 \pm 0.02$ & $0.80 \pm 0.03$ & $-0.05 \pm 0.02$ & 35,8 & $0.03,0.02$ & {$[\mathrm{c}][4]$} & 0.81 & 4.45 \\
\hline HD 81040 & $5692 \pm 58$ & $4.36 \pm 0.05$ & $0.73 \pm 0.09$ & $-0.01 \pm 0.08$ & 38,12 & $0.07,0.06$ & [3] & 1.01 & 4.55 \\
\hline HD 89307 & $5967 \pm 32$ & $4.51 \pm 0.01$ & $1.33 \pm 0.08$ & $-0.13 \pm 0.04$ & 33,14 & $0.03,0.03$ & [2] & 1.01 & 4.42 \\
\hline HD 102195 & $5291 \pm 34$ & $4.45 \pm 0.04$ & $0.89 \pm 0.05$ & $0.05 \pm 0.05$ & 36,10 & $0.04,0.04$ & [4] & 0.86 & 4.56 \\
\hline HD 109749 & $5899 \pm 49$ & $4.31 \pm 0.03$ & $1.13 \pm 0.06$ & $0.32 \pm 0.06$ & 39,12 & $0.06,0.05$ & [3] & 1.15 & 4.31 \\
\hline HD 149143 & $6018 \pm 56$ & $4.31 \pm 0.04$ & $1.12 \pm 0.07$ & $0.45 \pm 0.07$ & 37,11 & $0.06,0.05$ & [3] & 1.26 & 4.26 \\
\hline HD 189733 & $5051 \pm 47$ & $4.53 \pm 0.08$ & $0.95 \pm 0.07$ & $-0.03 \pm 0.05$ & 37,7 & $0.05,0.05$ & {$[\mathrm{~d}][3]$} & 0.79 & 4.60 \\
\hline HD 196885 & $6340 \pm 39$ & $4.46 \pm 0.02$ & $1.51 \pm 0.07$ & $0.29 \pm 0.05$ & 34,16 & $0.04,0.07$ & [2] & 1.33 & 4.35 \\
\hline HD 212301 & $6254 \pm 29$ & $4.52 \pm 0.01$ & $1.43 \pm 0.06$ & $0.18 \pm 0.04$ & 36,9 & $0.03,0.01$ & {$[\mathrm{e}][4]$} & 1.27 & 4.45 \\
\hline
\end{tabular}

$\dagger$ The instruments used to obtain the spectra were: [1] 2.2-m ESO/FEROS, [2] TNG/SARG, [3] 1.2-m Swiss Telescope/CORALIE, [4] 3.6-m ESO/HARPS;

HD 34445 and HD 196885 have been recently reported as new planet-hosts, although we are not aware of the existence of a discovery paper. They are included in the exoplanets Encyclopedia: http://vo.obspm.fr/exoplanetes/encyclo/catalog-main.php. The rest of the stars had already been reported in publication or already been submitted. For some stars, the stellar parameters have been published in the planet discovery paper: [a]-Udry et al. (2006), [b]-Santos et al. (2004), [c]- Lovis et al. (2006), [d]-Bouchy et al. (2005), [e]-Lo Curto et al. (2006).

(in $\mathrm{m} \AA$ ). The points are all very close to the identity line. We note a slightly better result for the less line-populated region of the spectrum, as expected.

In Fig. 5 we illustrate how the values displayed in the box in Fig. 4 change when fixing resolution and varying the signal to noise ratio. We can see the slope is always very close to 1 , meaning that there is good agreement between the two measurements. The results are generally very good except for the spectra with high noise level where it becomes more difficult to identify the lines. Even for low resolutions, the results are still reasonable with good typical observational values of signal to noise ratio. We note, however, that increasing the resolution decreases the mean difference of $E W$. This result may be from DAOSPEC taking false lines generated by the noise. Since DAOSPEC subtracts the identified lines in each loop of the code from the spectrum, the value for the strength of real lines will be underestimated, thus providing there lower values for the $E W$ measurement. When the noise is high, it may identify those false lines that are removing strength to the real lines.

We have also tested setting the signal to noise ratio $\sim 100$ and varying the resolution. The results show similar behavior to the ones presented in Fig. 5; i.e. the slope of the linear fit of the points is also very close to 1 , and almost all lines are identified until we reach very low values for the spectral resolution $(R \sim 10000)$. The results are always better in the less line-populated regions of the spectrum for the different kinds of spectra, as expected. For instance, the values for the rms and mean difference presented in 4 are higher in the $4500 \AA$ region when compared to those found for the $6000 \AA$ region. The number of identified lines in both cases are high. Only 2 lines in each region were not found by DAOSPEC for this spectral resolution and noise level.

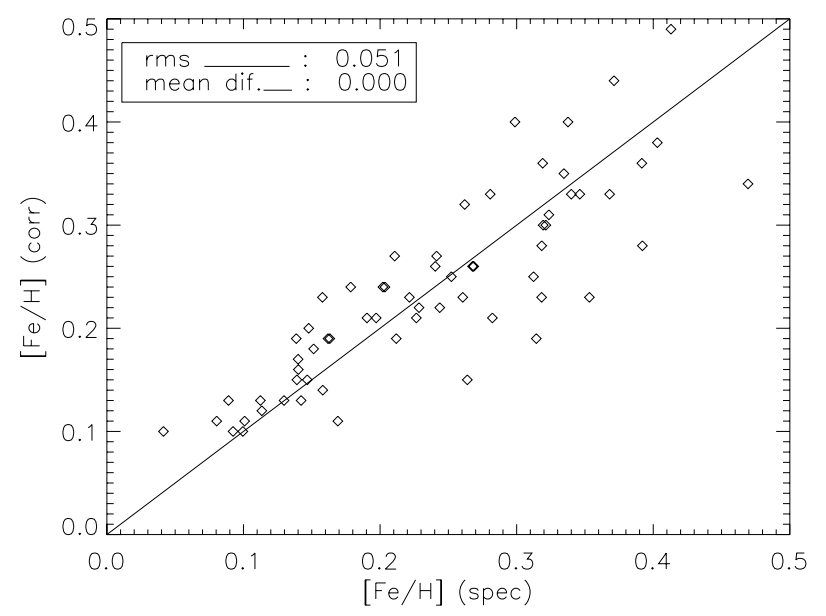

Fig. 3. Comparison of the spectroscopic and correlation function metallicities of our program stars for the stars presented in this paper. The solid line represents a 1:1 relation.

\subsection{Testing with real data}

DAOSPEC seems to work well with different sets of resolutions and even with high noise levels. In this section we describe the results obtained by using DAOSPEC on our sample of stars with FEROS spectra. We used the same line list as before.

One of the parameters of DAOSPEC is the spectral wavelength interval for the continuum fitting and the $E W$ measurements. Using the full individual spectrum interval, we got a large dispersion on the measurements. This result may come from the bad fitting of the continuum level to the large wavelength interval used in the computation. To fix this problem, we used smaller intervals of $500 \AA, 250 \AA$, and $100 \AA$ wide, at a time, 

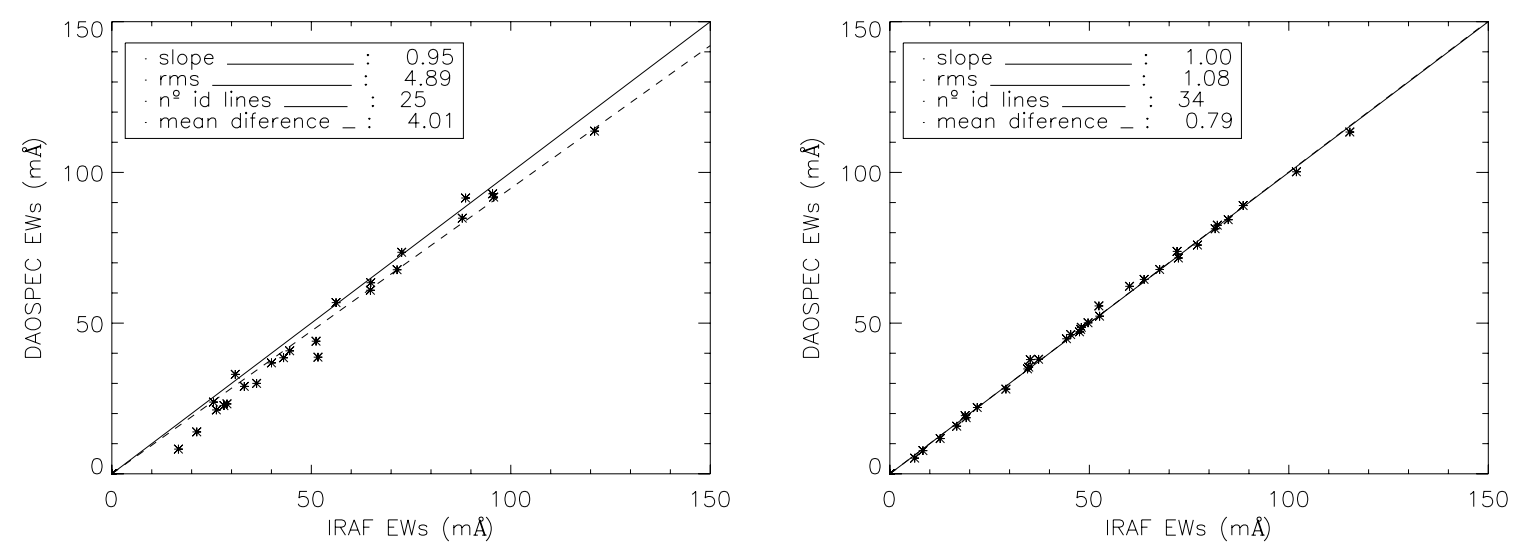

Fig. 4. Comparison of DAOSPEC and "hand made" measurements in two different regions of the spectra for $S / N \sim 100$ and $R \sim 50000$. Filled line: identity line; dashed line: linear fit; left: [4400-4650 ̊]; right: [6000-6300 ̊].
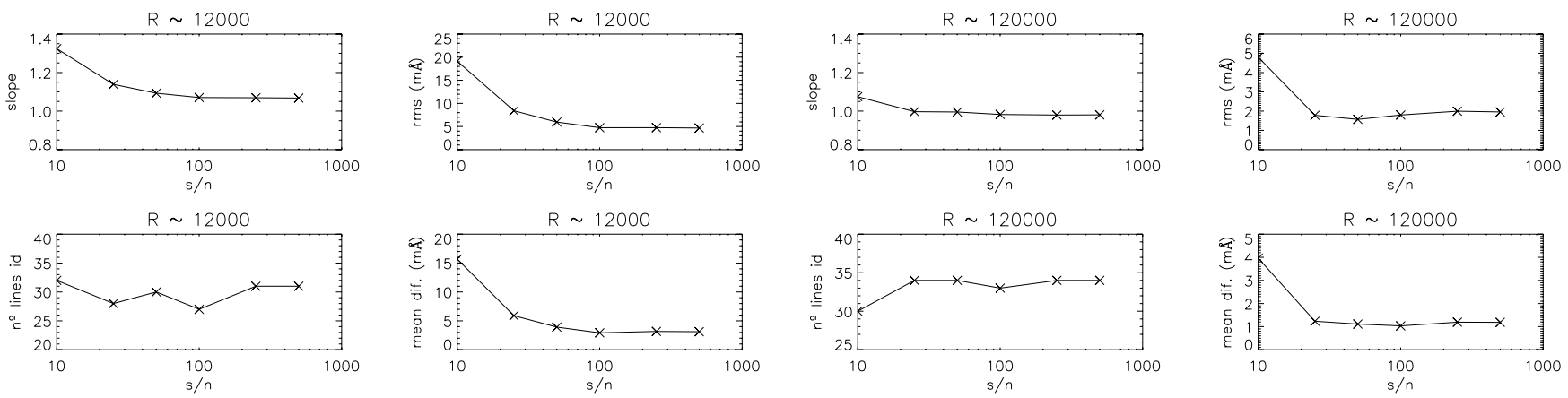

Fig. 5. Variation in the rms, slope, number of lines, and mean difference seen in Fig. 4 when fixing resolution $(R \sim 12000$ and $R \sim 120000)$ and varying the noise level for the [6000-6300 ̊] spectral region.
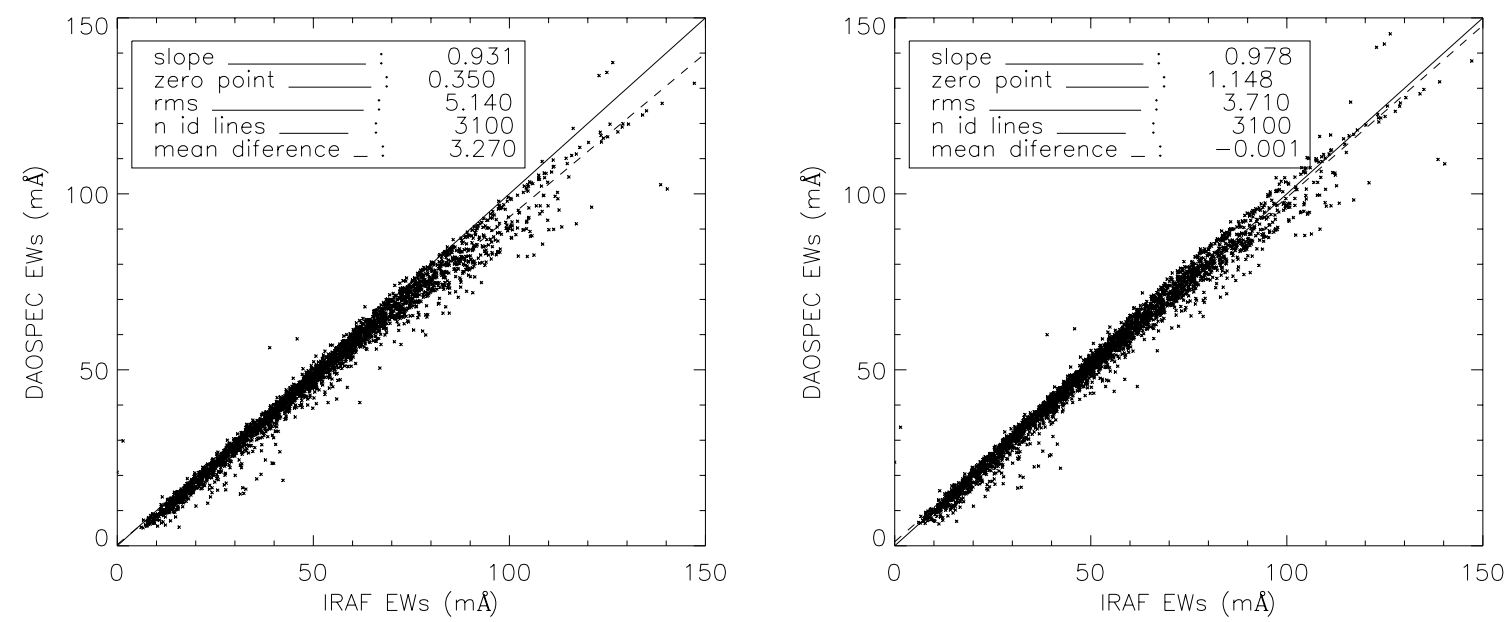

Fig. 6. Comparison of DAOSPEC and "hand made" measurements of $E W$ for 3100 lines in a sample of FEROS spectra. On the left we compare the direct DAOSPEC results with the "hand" made measurements. On the right, we compare the calibrated DAOSPEC results with the "hand" made measurements. See text for more details.

for the computation, making it easier to obtain the correct fit to the continuum level. The results for all stars improved considerably. The derived $E W$ can be seen in the left panel of Fig. 6 when using $100 \AA$ wavelength intervals. We can see that most of the points lie near the identity line. However, there seems to be a small underestimate of the measurements made by DAOSPEC with a mean value of about $3 \mathrm{~m} \AA$. When using smaller wavelength intervals, we obtain only slightly better results in terms of the dispersion. Considering our results, we recommend the use of $100 \AA$ intervals for the measurements. 
Other DAOSPEC parameters also deserve some particular comment $^{5}$. One of them is the order of the Legendre polynomial that fits the continuum level in the spectra, for which we used order 8 . For the value controlling the relative core flux we used a value of 0 . It is mentioned in the "DAOSPEC cookbook" that this parameter can be important for measuring strong and saturated lines (>200 mÅ). In our case we did not consider these cases because our lines are all weaker than $200 \mathrm{~m} \AA$ (the majority being weaker than $100 \mathrm{~m} \AA$ ). In what concerns the parameter that controls the smallest $E W$ for the lines, we used the default value of $5 \mathrm{~mA}$; i.e., DAOSPEC will not show lines with strengths weaker than $5 \mathrm{~m} \AA$. The initial guess for the FWHM resolution of the spectrum was taken as 14 pixels (typical in our case). This value was not fixed during the computation.

We tried to use the $E W$ derived using DAOSPEC for our set of Fe I and Fe II lines to derive stellar parameters. Preliminary results give us some offsets when compared with the ones determined in the regular way. Mean differences for the stellar parameters showed $\Delta T_{\mathrm{eff}} \sim-50 \mathrm{~K}, \Delta \log g \sim 0.03 \mathrm{dex}, \Delta \xi_{\mathrm{t}} \sim$ $-0.06 \mathrm{~km} \mathrm{~s}^{-1}$, and $\Delta[\mathrm{Fe} / \mathrm{H}] \sim-0.07$ dex. These significant offsets must be due to the small offset presented by the $E W$ derived using DAOSPEC.

\subsection{Calibrating DAOSPEC results}

In this section we present a calibration that we can use to correct for the small offset in $E W$ observed between DAOSPEC and the "hand made" measurements. The measurements of $E W$ can be dependent on several aspects. First, we expect different responses for different types of stars. We are focusing on F, G, and $\mathrm{K}$ stars, therefore the spectra can change considerably with the different effective temperatures. Another important cause of a different reaction is the local density of lines in the spectrum as a function of the wavelength, as seen in Fig. 4. Finally, a constant offset is clearly present, probably due to the way $E W$ are measured with DAOSPEC.

Given the above measurement dependences we calibrated the DAOSPEC $E W$ using the linear fit:

$$
\begin{aligned}
E W_{\text {cor }}= & 5.835+1.020 \times E W_{\text {dao }}+11.391(B-V)_{i} \\
& +(-0.002) \lambda_{i}
\end{aligned}
$$

where for each $i$ th line we have the calibrated value, $E W_{\text {cor }}$, the DAOSPEC measured $E W, E W_{\text {dao }}$, the respective $(B-V)$ value for the star that produced the line, $(B-V)_{i}$, and its wavelength $\lambda_{i}$. The $B-V$ is used here as a proxy for effective temperature.

After applying this calibration to the DAOSPEC $E W$ we compared the results with the "hand made" values in Fig. 6. The stars used in this comparison have values of $(B-V)$ ranging from 0.5 to 1.2 . We have a few stars that have lower temperature and for which measured $E W$ can be less reliable. By taking these stars out of the comparison, the rms presented in the right panel of Fig. 6 is reduced to $2.7 \mathrm{~m} \AA$ for $\sim 94 \%$ of the stars.

We note that since our sample is basically composed of stars with high metal content, this calibration may only be valid for metal rich stars. We expect that this calibration may change slightly with metallicity due to the intrinsic difference of the spectra; high metal-content spectra have more lines than low metal-content spectra, making it harder to determine accurate

\footnotetext{
${ }^{5}$ For details on the input variables of DAOSPEC please see the "DAOSPEC's Cookbook" "manual" that is available on the DAOSPEC webpage: http://www.bo.astro.it/ GC/personal/ epancino/projects/daospec.html
}

measurements. This fact must be verified with a sample of low metal-content stars in the future.

\section{Conclusions}

In this work we have presented accurate stellar parameters for a sample of 64 high metal-content stars. We have also presented metallicities and stellar parameters for 17 planet-host stars, most of which were not studied in our previous series of papers (Papers I-V). Adding these new 14 stars, we count a total sample of 133 planet-host stars with high accuracy and uniform stellar parameters. This list is available at http:// astro.oal.ul.pt/ nuno/table_star_planets.dat

As in previous works, these two samples of solar type stars were analyzed using the same method and model atmospheres to determine stellar parameters and $[\mathrm{Fe} / \mathrm{H}]$. Therefore the samples are uniform, unique, and appropriate for future studies of stellar metallicity-giant planet connection.

We present the results of a new code that we expect to use to measure $E W$ for the stellar parameter determination. We show that we can use DAOSPEC (Pancino \& Stetson 2006) to automatically measure $E W$. However, one has to be careful in how to use it. A significant increase in the number of iron lines in the line list used for the parameter determination may be needed in order to have a high statistical strength in the results.

Using higher-resolution spectra such as the ones collected by HARPS (Mayor et al. 2003), we should obtain better results. Another possible improvement for the results of an automatic determination of stellar parameter is a possible, fully relative analysis, i.e. using DAOSPEC to obtain the $E W$ measurements in order to measure the $E W$ in the solar spectrum.

Acknowledgements. S.G.S and N.C.S. would like to thank the support from Fundação para a Ciência e Tecnologia (Portugal) in the form of two fellowships and grants POCI/CTE-AST/56453/2004 and POCTI/CTE-AST/57610/2004 from POCTI, with funds from the European program FEDER.

\section{References}

Beirão, P., Santos, N. C., Israelian, G., \& Mayor, M. 2005, A\&A, 438, 251 Bodaghee, A., Santos, N. C., Israelian, G., \& Mayor, M. 2003, A\&A, 404, 715 Bouchy, F., Udry, S., Mayor, M., et al. 2005, A\&A, 444, L15

Ecuvillon, A., Israelian, G., Santos, N. C., et al. 2004a, A\&A, 418, 703

Ecuvillon, A., Israelian, G., Santos, N. C., et al. 2004b, A\&A, 426, 619

Ecuvillon, A., Israelian, G., Santos, N. C., Mayor, M., \& Gilli, G. 2006a, A\&A, 449, 809

Ecuvillon, A., Israelian, G., Santos, N. C., et al. 2006b, A\&A, 445, 633

ESA 1997, The Hipparcos and Tycho Catalogues, 1239, ESA

Flower, P. J. 1996, ApJ, 469, 355

Gaidos, E. J. 2000, Icarus, 145, 637

Gilli, G., Israelian, G., Ecuvillon, A., Santos, N. C., \& Mayor, M. 2006, A\&A, 449,723

Gonzalez, G. 1997, MNRAS, 285, 403

Gonzalez, G. 1998, A\&A, 334, 221

Gonzalez, G. 2006, MNRAS, 367, L37

Gonzalez, G., \& Lambert, D. L. 1996, AJ, 111, 424

Guillot, T., Santos, N. C., Pont, F., et al. 2006, A\&A, 453, L21

Hubeny, I., Lanz, T., \& Jeffery, C. 1994, TLUSTY \& SYNSPEC - A user's guide Israelian, G., Santos, N. C., Mayor, M., \& Rebolo, R. 2001, Nature, 411, 163

Israelian, G., Santos, N. C., Mayor, M., \& Rebolo, R. 2003, A\&A, 405, 753

James, D. J., Melo, C., Santos, N. C., \& Bouvier, J. 2006, A\&A, 446, 971

Kurucz, R. 1993, ATLAS9 Stellar Atmosphere Programs and $2 \mathrm{~km} \mathrm{~s}^{-1}$ grid. Kurucz CD-ROM No. 13, Cambridge, Mass.: Smithsonian Astrophysical Observatory, 1993., 13

Laws, C., Gonzalez, G., Walker, K. M., et al. 2003, AJ, 125, 2664

Lo Curto, G., Mayor, M., Clausen, J. V., et al. 2006, A\&A, 451, 345

Lovis, C., Mayor, M., Pepe, F., et al. 2006, Nature, 441, 305

Mayor, M., Pepe, F., Queloz, D., et al. 2003, The Messenger, 114, 20

Pancino, E., \& Stetson, P. B. 2006, in preparation 
Pinsonneault, M. H., DePoy, D. L., \& Coffee, M. 2001, ApJ, 556, L59

Press, W. H., Teukolsky, S. A., Vetterling, W. T., \& Flannery, B. P. 1992, Numerical recipes in FORTRAN. The art of scientific computing (Cambridge: University Press, |c1992, 2nd ed.)

Robinson, S., Laughlin, G., Bodenheimer, P., \& Ficher, D. 2006, Arxiv, 000, in press

Santos, N. C., Israelian, G., \& Mayor, M. 2000, A\&A, 363, 228

Santos, N. C., Israelian, G., \& Mayor, M. 2001, A\&A, 373, 1019

Santos, N. C., Mayor, M., Naef, D., et al. 2002, A\&A, 392, 215

Santos, N. C., Israelian, G., Mayor, M., Rebolo, R., \& Udry, S. 2003, A\&A, 398, 363

Santos, N. C., Israelian, G., \& Mayor, M. 2004, A\&A, 415, 1153

Santos, N. C., Israelian, G., Mayor, M., et al. 2005, A\&A, 437, 1127
Santos, N. C., Pont, F., Melo, C., et al. 2006, A\&A, 450, 825

Schaerer, D., Charbonnel, C., Meynet, G., Maeder, A., \& Schaller, G. 1993a, A\&AS, 102, 339

Schaerer, D., Meynet, G., Maeder, A., \& Schaller, G. 1993b, A\&AS, 98, 523

Schaller, G., Schaerer, D., Meynet, G., \& Maeder, A. 1992, A\&AS, 96, 269

Sneden, C. 1973, Ph.D. Thesis, Univ. of Texas

Sozzetti, A. 2004, MNRAS, 354, 1194

Sozzetti, A., Yong, D., Carney, B. W., et al. 2006, AJ, 131, 2274

Santos, N. C., Mayor, M., Naef, D., et al. 2002, A\&A, 392, 215

Udry, S., Mayor, M., Naef, D., et al. 2000, A\&A, 356, 590

Udry, S., Mayor, M., Benz, W., et al. 2006, A\&A, 447, 361

Vauclair, S. 2004, ApJ, 605, 874 